\title{
Editorial
}

\section{One more wheel for a processing machine}

\author{
Y Ben-Neriah ${ }^{\star 1}$
}

Cell Death and Differentiation (2015) 22, 1235-1236; doi:10.1038/cdd.2015.71

The transcription factor NF- $k B$ is the major conductor of inflammatory and immune responses and ever since its discovery has attracted an immense interest mainly due to its common disease involvement. ${ }^{1} \mathrm{NF}-\kappa \mathrm{B}$ activation and the ubiquitin-proteasome system have a special affiliation, and targeting the cellular NF- $\kappa \mathrm{B}$ inhibitor $\mathrm{I}_{\kappa \mathrm{B}}$ for ubiquitndependent proteasomal degradation was perhaps the first example of signal transduction via the ubiquitin-proteasome system. ${ }^{2}$ Whereas the most studied ubiquitination-controlled proteolysis step in NF- $\kappa$ B activation is the degradation of the $\mathrm{I}_{\kappa}$ $\mathrm{Bs},{ }^{3}$ the first evidence for proteasomal degradation in the NF$\kappa \mathrm{B}$ pathway was in fact observed in the processing (i.e., partial degradation for producing a functional polypeptide) of the NF$\kappa \mathrm{B}$ precursors into mature proteins. ${ }^{4}$ The two precursor proteins, NF- $\kappa$ B1 p105 and NF- $\kappa$ B2 p100, by way of their ankyrin repeats, also function as $I_{\kappa} \mathrm{Bs}$, withholding NF- $\kappa \mathrm{B}$ from the chromatin, but exactly how they integrate as $I_{k} B s$ into the canonical NF- $k B$ activation pathway remains mostly obscure. Processing of the NF- $k B$ precursors is facilitated by cellular stimuli that activate NF- $\kappa$ B. p100 is mostly processed following IKKa phosphorylation, which is activated by NF- $\kappa \mathrm{B}$-inducing kinase (NIK) as part of the non-canonical NF- $\kappa B$ signaling pathway. p105 is, however, primarily processed constitutively, but the processing rate is enhanced in stimulated cells by canonical IKK. Whereas p100 processing appears to be mediated by the E3 ubiquitin ligase $\beta$-TrCP, which targets all the IkBs, signal-induced interaction of $\beta$-TrCP with p105 results in its degradation rather than processing. Therefore, it has been debated for a long time if generation of the mature NF- $\kappa \mathrm{B}$ p50 subunits is mainly due to constitutive proteasomal processing of $\mathrm{p} 105$, either with or without ubiquitination, or perhaps it is achieved by other means, such as contranslational biogenesis. ${ }^{5}$ This debate may be resolved soon by a discovery made now by Ciechanover and colleagues in identifying KPC1 as the E3 ligase that facilitates p105 processing via ubiquitination. ${ }^{6}$ Using once more the legendary rabbit reticulocyte lysate by which Ciechanover et al. ${ }^{7}$ originally discovered the ubiquitin-proteasome system, biochemical fractionation identified the long sought out p105 E3. In addition to a fundamentally important discovery, this successful undertaking proves that traditional biochemistry has still a respectable room in pursuing cellular processes.
KPC1 has been identified before as a component of an E3 ligase that targets $p 27^{\text {Kip1 }}$ for ubiquitin degradation at an early cell cycle phase. ${ }^{8}$ p27 is a cell cycle inhibitor and RNAi of KPC1 delays p27 degradation and suppresses cell growth rate. It would have therefore been expected that KPC1 overexpression would support tumor growth due to enhanced p27 degradation. On the contrary, Kravtsova-Ivantsiv et al. show that KPC1 overexpression in cancer cell lines suppresses tumor growth. This is evident both in anchorageindependent growth assays in tissue culture, as well as in xenograft studies in mice. Tumor growth suppression correlates with nuclear abundance of p50 in KPC1-overexpressing xenografts, a correlation that is also observed in samples of several human tumors by immunohistochemistry. Furthermore, overexpression of the NF- $\kappa \mathrm{B}$ p50 subunit has a growth suppression effect similar to KPC1. It therefore seems that in tumor growth, KPC1 has a crucial role in NF- $K B$ processing, overpowering its role in cell cycle regulation. Indeed, there was a noticeable effect of KPC1 overexpression on the proliferation rates of the tumor cells as monitored by Ki-67 staining, indicating that the regulation of $\mathrm{NF}-\kappa \mathrm{B}$ or other $\mathrm{KPC} 1$ substrates overrules the expected p27 stabilization effects, which by itself was not evident in the tumor cells studied. Kravtsova-Ivantsiv et al. implicate p105 processing in the tumor suppression effects of KPC1 and propose that the nuclear accumulation of p50 functions as a suppressor of the pro-tumorigenic effects of NF- $\kappa \mathrm{B}$, pointing at certain NF- $\kappa \mathrm{B}$ target genes that might be affected by p50 accumulation in glioblastoma cells.

Whereas the identification of KPC1 as the E3 responsible for $\mathrm{p} 105$ processing is an important advance in understanding the NF- $\kappa$ B pathway, we are left with several questions related to the regulation of $\mathrm{p} 105$ processing and its relationship to the other NF- $\kappa$ B precursor, p100. Kravtsova-Ivantsiv et al. show that the interaction of KPC1 with p105 and subsequent p105 ubiquitination is enhanced by IKK phosphorylation, which also facilitates the interaction of p105 with its other E3, $\beta$-TrCP, which leads to precursor degradation. Is the nature of ubiquitination facilitated by the two E3s distinct enough to account for the different effects of the proteasome, processing versus degradation? Previous work from the Ciechanover lab implicated multi-monoubiquitination in $\mathrm{p} 105$ processing rather than conventional polyubiquitination; ${ }^{9}$ is it therefore possible that while polyubiquitination instructs proteasomal degradation, monoubiquitination promotes proteasomal processing? Related to this is the question of competing E3 functions: how are the two E3s coordinated to distinguish processing from

\footnotetext{
${ }^{1}$ Lautenberg Center for Immunology and Cancer Research, Hebrew University-Hadassah Medical School, Jerusalem, Israel

*Corresponding author: Y Ben-Neriah, Lautenberg Center for Immunology and Cancer Research, Hebrew University-Hadassah Medical School, Jerusalem 9112102 , Israel. Tel: +972 26758718; Fax: +972 26430834; E-mail: yinonb@ekmd.huji.ac.il
} 


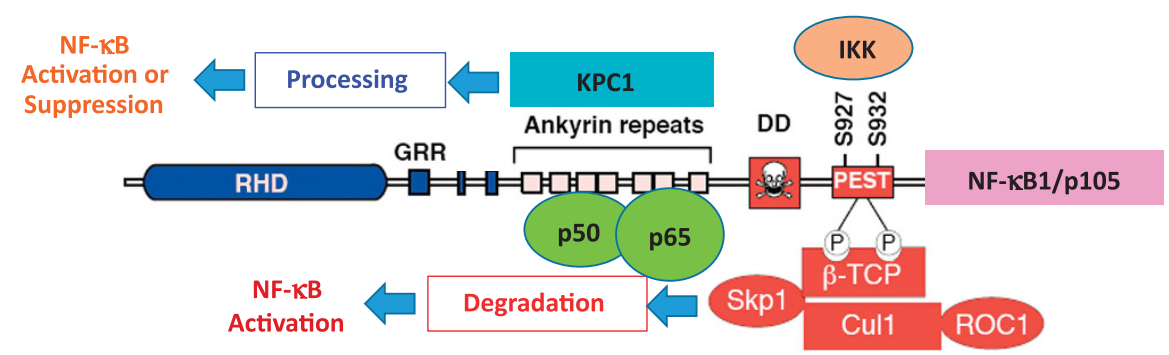

Figure $1 \mathrm{NF}-\kappa \mathrm{B} 1 / \mathrm{p} 105$ fate determination by two E3s: $\beta$-TrCP binding to the IKK-phosphorylated degron at the C-terminal PEST domain facilitates full proteasomal degradation, whereas KPC1 binding to the ankyrin repeat domain facilitates processing — partial proteasomal proteolysis that generates $\mathrm{p50}$, the mature NF- $\kappa \mathrm{B}$ component. The fate of NF- $\mathrm{KB} 1 / \mathrm{p} 105$ could be dictated by competition between KPC1 and Rel proteins (p50 and p65) for binding the p105 ankyrin repeat: KPC1 binding (top) will promote processing, whereas Rel binding (bottom) would either preserve the intact precursor, or possibly promote $\beta$-TrCP-mediated degradation. In addition, the observation of KravtsovaIvantsiv et al. that IKK phosphorylation facilitates KPC1-mediated p105 ubiquitination, similarly to the well-recognized IKK-dependent $\beta$-TrCP-mediated p105 ubiquitination, may also indicate a cooperation between the two E3s in p105 processing. RHD, Rel homology domain; GRR, glycine rich region; DD, death domain; PEST sequence is a peptide sequence that is rich in proline $(P)$, glutamic acid $(E)$, serine $(S)$ and threonine $(T)$.

degradation? Is it possible for example that some p105 interacting proteins promote association with a particular E3? In this manner, naked, newly synthesized p105 could perhaps be preferentially targeted by KPC1 for processing, while bound p105, functioning as a RelA/p50 inhibitor, would be targeted by $\beta$-TrCP for degradation (Figure 1).

The study of Kravtsova-Ivantsiv et al. opens up another interesting issue concerning the mode of processing of the other NF-KB precursor- $\mathrm{p} 100$; is $\beta$-TrCP sufficient to mediate p100 processing, or does it require another specialized E3? Related to this is a recent observation by Scheidereit et al., showing that the two NF-KB precursors are often physically associated, and that lymphotoxin stimulation, which induces phosphorylation and subsequent processing of $\mathrm{p} 100$, results in concurrent processing of $\mathrm{p} 105 .{ }^{10} \mathrm{It}$ remains to find out then which E3 is required for lymphotoxin-induced precursor coprocessing, $\mathrm{KPC} 1, \beta-\mathrm{TrCP}$ or both. Interestingly, there is a human autosomal dominant mutation in the $\beta$-TrCP recognition degron of $\mathrm{p} 100$ that results in a B-cell immunodeficiency syndrome, displaying defects both in the non-canonical and canonical NF-kB pathway, ${ }^{11}$ possibly supporting a common processing mechanism for the two NF-kB precursors.

Finally, identification of KPC1 as a new signaling component in the NF- $\kappa$ B activation pathway may provide new therapeutic opportunities, especially in the fields of inflammation and cancer, yet pharmacological enhancement of any E3 function remains particularly challenging.

\section{Conflict of Interest}

The author declares no conflict of interest.

Acknowledgements. Work from the author laboratory is as supported by grants from Israel Science Foundation (ISF)-Centers of Excellence, the European Research Council (294390 PICHO), Dr. Miriam and Sheldon G. Adelson Medical Research Foundation (AMRF) and Israel Cancer Research Fund.

1. Hayden MS, Ghosh S. Genes Dev 2012; 26: 203-234.

2. Karin M, Ben-Neriah Y. Annu Rev Immunol 2000; 18: 621-663.

3. Kanarek N, Ben-Neriah Y. Immunol Rev 2012; 246: 77-94.

4. Fan CM, Maniatis T. Nature 1991; 354: 395-398.

5. Lin L, DeMartino GN, Greene WC. Cell 1998; 92: 819-828.

6. Kravtsova-Ivantsiv $Y$ et al. Cell 2015; 161: 333-347.

7. Ciechanover A, Hod Y, Hershko A. Biochem Biophys Res Commun 2012; 425: 565-570.

8. Kamura T et al. Nat Cell Biol 2004; 6: 1229-1235.

9. Kravtsova-Ivantsiv Y, Cohen S, Ciechanover A. Mol Cell 2009; 33: 496-504.

10. Yilmaz ZB et al. Cell Rep 2014; 9: 1756-1769.

11. Lee CE et al. Blood 2014; 124: 2964-2972. 